\title{
Cardiac regeneration by pharmacologically active microcarriers releasing growth factors and/or transporting adipose-derived stem cells
}

\author{
Monia Savi, ${ }^{1,2}$ Leonardo Bocchi, ${ }^{1,3}$ Emanuela Fiumana, ${ }^{3,4}$ Caterina Frati, ${ }^{5}$ \\ Francesca Bonafé, 3,4 Stefano Cavalli, ${ }^{5}$ Paolo Giovanni Morselli, 6 Jean-Pierre Karam,4,7 \\ Claudia Montero-Menei,7 Claudio Marcello Caldarera,3,4 Carlo Guarnieri,3,4 \\ Claudio Muscari, 3,4 Donatella Stilli,,3 Federico Quaini, 2,3 Ezio Musso, 1,3 \\ ${ }^{1}$ Department of Biosciences, University of Parma; ${ }^{2}$ Department of Clinical and Experimental \\ Medicine, University of Parma; ${ }^{3}$ National Institute of Cardiovascular Research - INRC, University \\ of Bologna; ${ }^{4}$ Department of Biochemistry, University of Bologna; ${ }^{5}$ Department of Biomedical, \\ Biotechnological and Translational Sciences, University of Parma; ${ }^{6}$ Department of Specialized \\ Surgery and Anesthetic Sciences, University of Bologna, Italy; ${ }^{7}$ INSERM U646, University of \\ Angers, France
}

\begin{abstract}
We tested the hypothesis that cardiac regeneration through local delivery of adipose-derived stem cells (ASCs), activation of resident cardiac stem cells via growth factors (GFs) [hepatocyte growth factor (HGF) and insulin-like growth factor 1 (IGF-1):GFs] or both, are improved by pharmacologically active microcarriers (PAMs) interacting with cells/molecules conveyed on their surface. Rats with onemonth old myocardial infarction were treated with ASCs, ASCs+PAMs, GF-releasing PAMs, ASCs+GF-releasing PAMs or vehicle. Two weeks later, hemodynamic function and inducibility of ventricular arrhythmias (VAs) were assessed. Eventually, the hearts were subjected to anatomical and immunohistochemical analyses. A significant ASCs engraftment and the largest improvement in cardiac mechanics occurred in ASC+GF-releasing PAM rats which by contrast were more vulnerable to VAs. Thus, PAMs may improve cell/GF-based cardiac regeneration although caution should be paid on the electrophysiological impact of their physical interaction with the myocardium.
\end{abstract}

Correspondence: Monia Savi, Department of Biosciences, University of Parma, Parco Area delle Scienze 11A, 43124 Parma, Italy.

Tel. +39.0521.902111 - Fax: +39.0521 .347038 .

E-mail: monia.savi@unipr.it

Key words: myocardial infarction, stem cell therapy, adipose-derived stem cells, growth factor, scaffold.

(C) Copyright M. Savi et al., 2014

Licensee PAGEPress, Italy

Journal of Biological Research 2014; 87:2141

doi:10.4081/jbr.2014.2141

This article is distributed under the terms of the Creative Commons Attribution Noncommercial License (by-nc 3.0) which permits any noncommercial use, distribution, and reproduction in any medium, provided the original author(s) and source are credited.

\section{Introduction}

In preclinical studies, cell transplantation has definitely emerged as a potential treatment strategy for heart failure secondary to myocardial infarction (MI), whereas data from large sample size clinical trials are conflicting. ${ }^{1}$ To ameliorate the unsatisfactory outcome of cell therapy in clinical settings, attention has been paid to several factors such as tailoring of cell type, fine tuning of procedural aspects, and optimization of engraftment techniques with the use of scaffolds. In recent years, several studies documented the existence in adipose tissue of stem cells [adipose-derived stem cells (ASCs)] which can be safely transplanted to an autologous or allogeneic host and differentiate into various cell types, including cardiomyocytes. ${ }^{2}$ Further, hepatocyte growth factor (HGF) and insulin-like growth factor-1 (IGF-1) (GFs) were shown to repair the infarcted heart and improve its electromechanical competence by respectively mobilizing resident cardiac stem cells (CSCs) and promoting their survival and proliferation. ${ }^{3}$ Finally, pharmacologically active microcarriers (PAMs) formed by biodegradable and non-cytotoxic poly (lactic-co-glycolic acid) microspheres which interact with cells/molecules conveyed on their surface and tissue microenvironment were proposed for enhancing cell engraftment in the injured myocardium. ${ }^{4}$ In the present study, we tested the hypothesis that stem cell based cardiac regeneration through intra-myocardial delivery of ASCs, GF-mediated local activation of CSCs, or both, can be improved by PAMs.

\section{Materials and Methods}

ASCs were isolated by human lipoaspirates. PAMs were coated with fibronectin and poly-lysine to improve cell adhesion and biological function. GFs were encapsulated into PAMs during the microsphere formulation. Thirty-eight adult male Wistar rats with one-month old MI were treated with ASCs (MI_ASC group, $\mathrm{n}=8$ ), ASCs+PAMs (MI_ASC+PAM, $\mathrm{n}=7$ ), GF-releasing PAMs (MI_PAM+GF, $\mathrm{n}=6$ ), ASCs+GF-releasing PAMs (MI_PAM+ASC+GF, $n=6$ ) or vehicle (MI_V, $\mathrm{n}=6$ ). Five additional rats were sham operated (SHAM). Two weeks after treatment, hemodynamic parameters were invasively determined in Millar conductance catheter studies and inducibility of ventricular 
a)

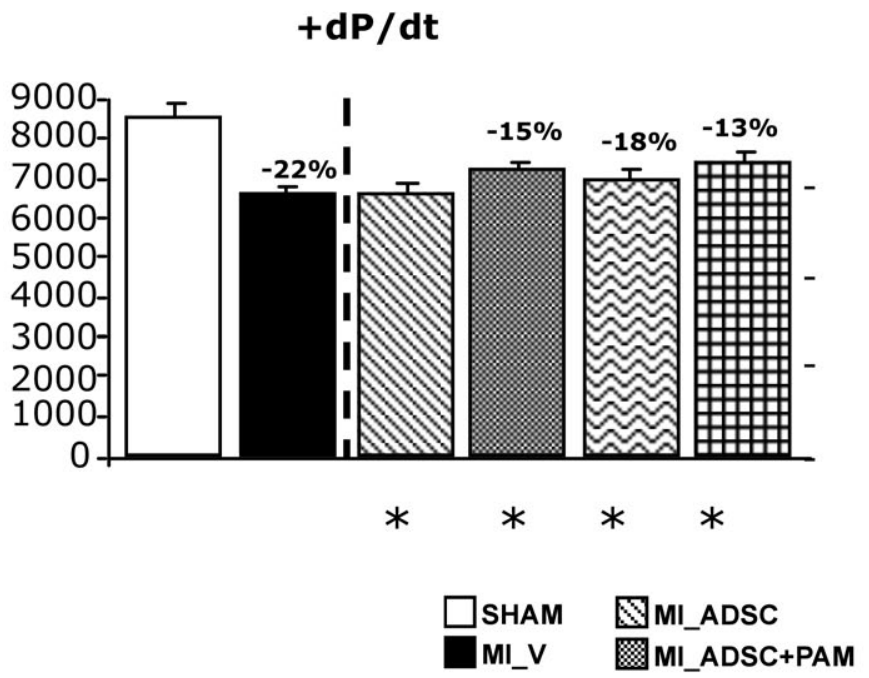

b)

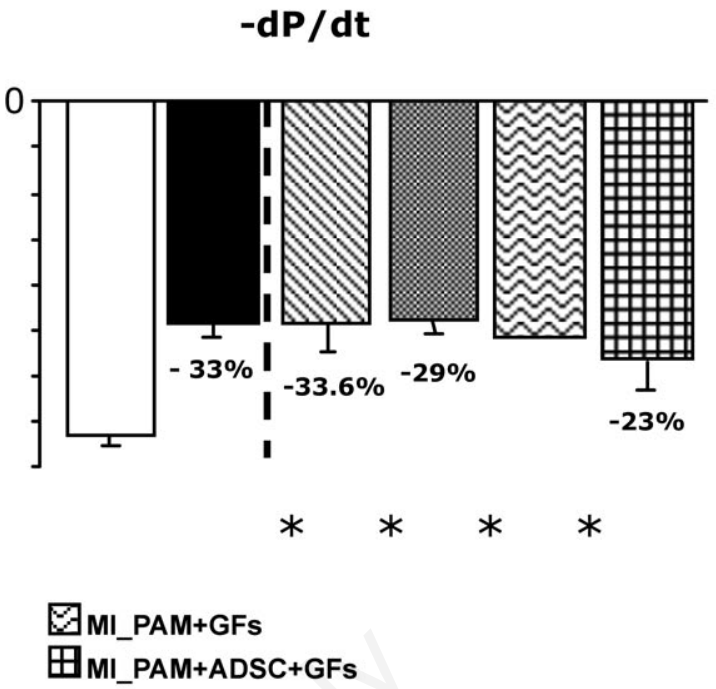

Figure 1. Mean values \pm standard error expressed in $\mathrm{mmHg} / \mathrm{s}$ of $+\mathrm{dP} / \mathrm{dt}$ (a) and $-\mathrm{dP} / \mathrm{dt}(\mathrm{b})$, measured in the 6 experimental groups. $* \mathbf{P}<0.05$, significant differences $v s$ sham operated rats.

arrhythmias was assessed during programmed stimulation in Langendorff-perfused hearts, by microelectrode array (MEA) system. Eventually, the hearts were subjected to anatomical, morphometric, and immunohistochemical analyses. ASC tracking was achieved by the detection at tissue level of both pre-labeled cells with Dil fluorochrome and human specific sex chromosomes by FISH.

\section{Results}

The deterioration of ventricular function in untreated MI rats was represented by an approximately $22 \%$ reduction in the maximal rate of ventricular pressure rise and decline $( \pm \mathrm{dP} / \mathrm{dt}$; Figure 1$)$ and a $35 \%$ prolongation of isovolumic contraction and relaxation times. $\mathrm{PAM}+\mathrm{ASC}+\mathrm{GF}$ was the most effective treatment leading to a substantial improvement of all hemodynamic parameters whose values approached those measured in SHAM rats (Figure 1).

The recovery of left ventricular (LV) hemodynamics in MI_PAM+ASC+GF group had a structural counterpart in a $10 \%$ reduction in $\mathrm{LV}$ chamber dilation together with a $20 \%$ increase in mass-to-chamber-volume ratio, thus cancelling out the unfavorable $\mathrm{LV}$ remodeling induced by MI (no significant difference $v s$ SHAM). In the same animals, a considerable amount of cell engraftment within the infarcted hearts was clearly documented by FISH immunohistochemical studies. Lastly, all treatments involving the use of PAM had beneficial effects on myocardial perfusion by significantly increasing the number of arterioles in the regenerated hearts. Again, the best results were obtained in the MI_PAM+ASC+GF group $(+75 \% ; \mathrm{P}<0.01$ vs MI-V).

By contrast, the improvement in cardiac mechanical function and structure mediated by $\mathrm{PAM}+\mathrm{ASC}+\mathrm{GF}$ treatment was not accompanied by a similar positive effect on electrical function. Indeed, arrhythmias occurring during programmed stimulation trended to be larger in all treated animals and more so in MI_PAM+ASC+GF group.

\section{Discussion}

PAMs appeared to improve ASC- or GF-based approaches to regenerate the infarcted heart and ameliorate cardiac function although caution should be paid on the electrophysiological impact of the physical interaction of microspheres with the myocardium.

\section{References}

1. Malliaras K, Marbán E. Cardiac cell therapy: where we've been, where we are, and where we should be headed. Brit Med Bull 2011;98:161-85.

2. Mizuno H, Tobita M, Uysal AC. Concise review: adipose-derived stem cells as a novel tool for future regenerative medicine. Stem Cells 2012;30:804-10.

3. Urbanek K, Rota M, Cascapera S, et al. Cardiac stem cells possess growth factor-receptor systems that after activation regenerate the infarcted myocardium, improving ventricular function and longterm survival. Circ Res 2005;97:663-73.

4. Karam JP, Muscari C, Montero-Menei CN. Combining adult stem cells and polymeric devices for tissue engineering in infarcted myocardium. Biomaterials 2012;33:5683-95. 